|| ISSN(online): 2589-8698 || ISSN(print): 2589-868X || International Journal of Medical and Biomedical Studies

Available Online at www.ijmbs.info

PubMed (National Library of Medicine ID: 101738825)

Index Copernicus Value 2018: 75.71

IJMBS

\title{
COMPARATIVE STUDY OF ENDOSCOPIC AND MICROSCOPIC ASSISTED MYRINGOPLASTY IN TIRUMALA HOSPITALS, KADAPA
}

\section{Dr. P. Surendra Babu}

MS (ENT), Consultant, Department of ENT, Tirumala Super speciality Hospital, Kadapa, AP

Article Info: Received 14 April 2019; Accepted 27 May. 2019

DOI: https://doi.org/10.32553/ijmbs.v3i5.1010

Corresponding author: Dr. P. Surendra Babu

Conflict of interest: No conflict of interest.

\section{Abstract}

Objective: The main aim of the study is to assess the operative time, graft uptake and audiological gain and post-operative scar in the group of the patients undergoing the endoscope assisted and the microscope assisted myringoplasty in Tirumala Hospital, Kadapa.

Material and Methods: Myringoplasty or type-1 tympanoplasty was performed by either the endoscope or the microscope assistance. Postaural approach was undertaken for microscope assisted myringoplasty. Underlay technique of myringoplasty was performed using temporalis fascia graft in all the patients suffering from the inactive mucosal chronic otitis media. Post operative evaluation was done in terms of operative time, graft uptake and audiological gain. The patients were followed up for a period of 6 months. The study was longitudinal in nature and comprised of 100 patients suffering from the chronic otitis media inactive mucosal disease. The patients are divided into two groups consisting of 50 in each group. One group of patients have undergone microscopic assisted myringoplasty one group undergone endoscopic assisted myringoplasty and were followed up regularly. Study period is one year from January 2016 to December 2016 attending outpatient otolaryngology department were included in the study.

Results: The operative time in the patients undergoing the endoscope assisted myringoplasty(EAM) was 45minutes whereas it was 60 minutes in the patients undergoing the microscope assisted myringoplasty(MAM). The tympanic membrane graft uptake and audiological gain was assessed at 1 month, 3months and 6 months post operatively. The successful closure of the tympanic membrane perforation was higher with the endoscope assisted myringoplasty as compared to the microscope assisted myringoplasty at 1 month, 3 months and 6 months of follow up. The mean audiological gain following myringoplasty with both the techniques used was noted. The mean audiological gain was found to be marginally better with the endoscope assisted myringoplasty at follow up of 1, 3 and 6 months as compared to the microscope assisted myringoplasty.

Conclusion: The operative time was less in the endoscope assisted technique as compared to the microscope assisted technique. The post operative morbidity (evaluated in terms of post operative scar) was less in the group of patients undergoing the Transcanal endoscope assisted as compared to microscope assisted myringoplasty. There was no significant difference in either graft uptake or post-operative audiological gain in the patients undergoing myringoplasty by endoscope assisted and microscope assisted technique. The endoscope assisted myringoplasty has advantages of better assessment of ossicular chain and in the patients with canal overhang it improves the visualisation of the margins of the perforation thus obviating the need for canalplasty. Thus the endoscope assisted myringoplasty can be used as a preferred alternative to the conventional microscope assisted myringoplasty, with better post operative results and reduced operating time and morbidity of the surgical procedure.

Keywords: Chronic otitis media (COM); Myringoplasty; Endoscope assisted myringoplasty; Microscope assisted myringoplasty.

\section{Introduction}

Background: To determine the advantages and disadvantages of endoscope as compared to microscope in myringoplasty surgery and to compare the results of both groups.

Tympanic membrane (TM) perforations are a common problem, most often as consequence of middle ear infection, traumatic rupture, or postoperative complication.

Despite the autoregenerative capacity of the eardrum, chronic perforations may be subject to surgical repair. ${ }^{1}$ The main goal of tympanoplasty (TP) is to restore anatomy and function and to eliminate disease; therefore, an uninterrupted TM, an 
aircontaining mucosal-lined middle ear and a secure connection between the TM and the inner ear fluids are essential. ${ }^{2,3}$ The introduction of endoscopy to otologic surgery was underpinned by the ability to provide better access and view to otherwise hidden areas of the middle ear, such as the retrotympanum, anterior epitympanum, or middle ear folds, resulting in better appreciation of their relationships. ${ }^{4}$ This improved visualization results from closeness of the light source to the surgical field and wide angle optics, thereby transforming the external auditory canal into an excellent surgical portal. ${ }^{5}$ The endoscopic revolution has also lead to advances in anatomic and pathophysiologic concepts, which elucidate the role that middle ear folds play in blocking middle ear ventilation routes in patients with chronic otitis media. A more conservative approach preserving the mastoid tissues decreases morbidity and may improve postoperative middle ear ventilation, owing to their role in middle ear gas exchange. Systematic intraoperative visualization, analysis, and in some cases removal of these folds should be regarded as essential to restore middle ear physiology.$^{6-8}$ Endoscopes better illustrate these findings and aid in engaging this new philosophical perspective. Endoscopic procedures, nevertheless, have several disadvantages. Given the diameter of the endoscope in relation to the ear canal, dissection may only be feasible with 1 hand and, thus, inefficient and challenging, chiefly when there is blood in the surgical field. Refinement of endoscopic skills and adopting precautionary hemostatic measures are paramount, a task more difficult to master by the surgeon with limited endoscopic training. Other caveats relate to heat dissipation and ototoxicity of antifog solutions. Concern over thermal injury is warranted for elevated temperatures may occur up to $8 \mathrm{mmfrom}$ the endoscope tip ${ }^{9}$; thus, smaller 3-mm endoscopes with submaximal light intensity $(<60 \%)$, frequent removing-repositioning, irrigation of the surgical field, and suction are recommended.

Chronic suppurative otitis media (CSOM) is a long standing infection of a part or whole of the middle ear cleft. CSOM is characterized by ear discharge, a permanent perforation, and impairment of hearing. Comparative study of microscopic-assisted and endoscopic-assisted myringoplasty lead to a new concept of the treatment of deafness secondary to chronic infection in the middle ear and mastoid and the new method was called "tympanoplasty."
Tympanoplasty is an operation to eradicate disease in the middle ear and to reconstruct the hearing mechanism, with or without tympanic membrane (TM) grafting." Myringoplasty is performed when there is TM perforation without any ossicular damage. This comparative study deals with anatomical and functional outcome in a series of patients who underwent endoscope-assisted myringoplasty (EAM) and microscope-assisted myringoplasty (MAM) with underlay technique using temporalis fascia graft.

\section{Materials and Methods}

The cases for this study were taken from the outpatient casesof the Department of Otorhinolaryngology, in Tirumala hospital, Kadapa during January 2016 to December 2016. The study comprised of 100 patients of COM, inactive mucosal disease of both the sexes and age group 15-45 year. Patients are divided into two groups. Group A consisted of 50 patients who underwent endoscope assisted myringoplasty and Group B also consist of 50 patients who underwent microscope assisted myringoplasty. In the endoscope assisted group Transcanal approach of myringoplasty was performed in all patients. In microscope assisted group all 50 patients underwent Postaural approach myringoplasty. The graft material used in all the procedures was temporalis fascia.

Inclusion criteria

Patient having small, medium, and large dry centralperforation of TM. Patient not having the evidence of active infection of nose, throat, and paranasal sinuses.

\section{Exclusion criteria}

Patients having hearing loss of sensorineural type or mixed type. Ossicular abnormality in preoperative or during surgery.

Patient with clinical and radiological evidence of atticoantral disease. Patients with a history of the previous surgery for chronic otitis media.

All the patients who presented signs and symptoms suggesting tubotympanic type CSOM were submitted to an assessment protocol, based on a guided history taking, specific physical exam (otoscopy), and subjected to audiogram

All the patients are followed after surgery as usual on the7th and 14th days. However, the audiogram was 
done on $1^{\text {st }}, 3^{\text {rd }}$ and $6^{\text {th }}$ month for hearing assessment.

Statistical analysis-

Data was analyzed by using SPSS 20 . Chi- square test was used for comparing graft uptake and unpaired ttest was used for statistical comparison of post operative audiological gain. $P$ value of $<0.05$ was considered significant while $p$ value $<0.01$ was considered highly significant.

RESULTS-

The study included 100 patients who underwent Myringoplasty/type-1 Tympanoplasty for chronic otitis media (inactive mucosal type). In our study of 100 patients 46 were male and 54 were female with a female to male ratio of 1.7:1.

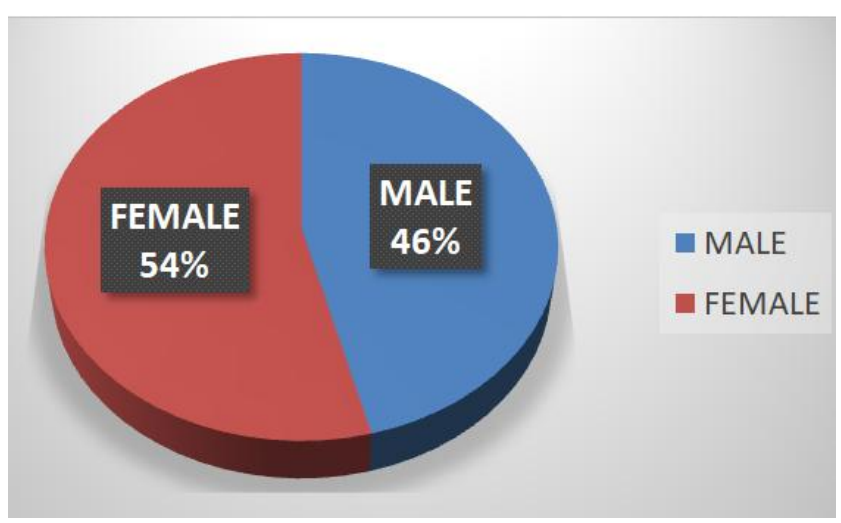

Figure 1: Sex distribution of patients

The age of the patient ranged $15-45$ years

Table 1: Age distribution of patients

\begin{tabular}{|c|c|c|}
\hline AGE GROUP & MAM & EAM \\
\hline $18-25$ rs & 23 & 21 \\
\hline $26-55$ rs & 16 & 13 \\
\hline $36-45$ rs & 11 & 16 \\
\hline TOTAL & 50 & 50 \\
\hline
\end{tabular}

\section{Postoperative tympanic membrane graft uptake.}

Evaluation of the tympanic membrane graft uptake following myringoplasty was done at 1 month, 3 months and 6 months post operatively. On otoscopic examination, successful uptake of the tympanic membrane graft was taken in the cases in which no remnant of perforation was present in any of the quadrant. The success rate of graft uptake for the endoscope assisted myringoplasty was $88.0 \%$ and for the microscope assisted group was $84.0 \%$ at one month post operatively. The tympanic membrane graft uptake at 1 month, 3 months and 6 months after the surgery for different techniques used with ' $p$ ' values are summarized in (Table 2 ).

Table 2: Post-operative graft uptake in follow up in both procedures

\begin{tabular}{|c|c|c|c|}
\hline & SUCCESS & FAILURE & \\
\hline \multicolumn{3}{|c|}{ 1Month } & \multirow{3}{*}{$p=0.564$} \\
\hline EAM & 44 & 6 & \\
\hline MAM & 42 & 8 & \\
\hline \multicolumn{3}{|c|}{3 Months } & \multirow{3}{*}{$p=0.766$} \\
\hline EAM & 43 & 7 & \\
\hline MAM & 44 & 6 & \\
\hline \multicolumn{3}{|c|}{ 6Months } & \multirow{3}{*}{$p=0.766$} \\
\hline EAM & 43 & 7 & \\
\hline MAM & 44 & 6 & \\
\hline
\end{tabular}

\section{EAM GRAFT STATUS}

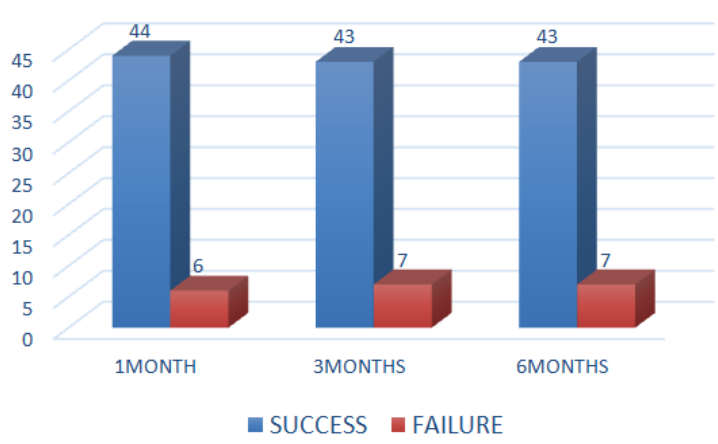

Figure 2:

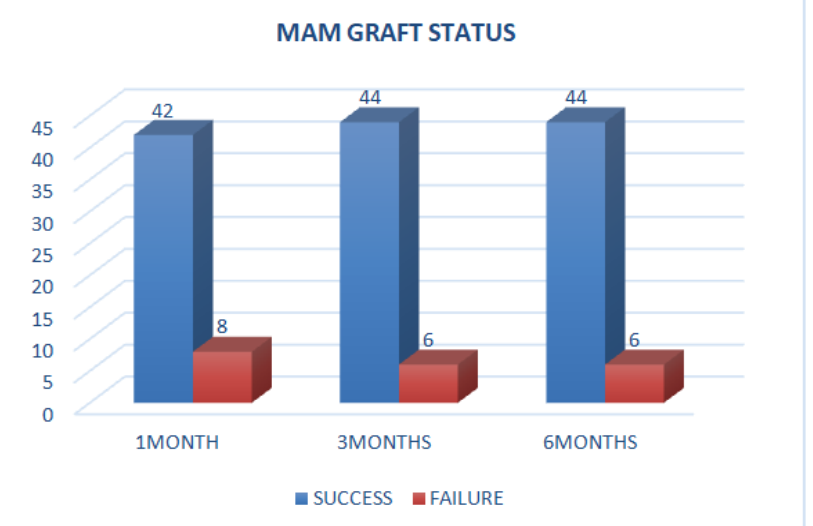

Figure 3:

\section{Post-operative audiological gain.}

In our study pure tone audiometry was done at 1 month, 3 months and 6 months postoperatively and audiological gain was evaluated. AB gap (air -bone conduction gap) per case was calculated as mean of $A B$ gap at four frequencies $(0.5,1,2 \& 4 \mathrm{KHz})$ pre operatively and post operatively. Audiological gain was calculated for each patient by subtracting the post op $A B$ gap from the pre op $A B$ gap. The mean 
was calculated for each variable by dividing the sum of audiological gain in that group by the total number of cases in the same group. Mean audiological gain at 1 month, 3 months and 6 months after the surgery for different techniques with statistical significance (' $p$ ' value) are summarized in (Table 3 ).

Table 3: Mean audiological gain in various techniques

\begin{tabular}{llllll}
\hline & PROCEDURE & $\begin{array}{l}\text { MEAN } \\
\text { GAIN }\end{array}$ & AUDIOLOGICAL & SD & $\begin{array}{l}\text { p } \\
\text { VALUE }\end{array}$ \\
\hline \multirow{2}{*}{ ONE MONTH } & EAM & 9.37 & 3.15 & 0.473 \\
\cline { 2 - 5 } & MAM & 8.89 & 3.57 & \\
\hline \multirow{2}{*}{ THREE } & EAM & 9.56 & 3.14 & 0.236 \\
\cline { 2 - 5 } MONTHS & MAM & 8.80 & 3.28 & \\
\hline SIX MONTHS & EAM & 9.79 & 2.95 & 0.329 \\
\cline { 2 - 4 } & MAM & 9.16 & 3.46 & \\
\hline
\end{tabular}

It was observed that EAM required an average $45 \mathrm{~min}$ (range of 40-60 $\mathrm{min}$ ) and MAM required an average of $60 \mathrm{~min}$ (range of 45-70 min).

Variations of external auditory canal such as stenosis, tortuosity, bony overhangs, etc., hamper the view of TM when visualized through microscope. Therefore, a need to manipulate the patients head or the microscope repeatedly to visualize all the parts of TM. Sometimes, in spite of manipulation, TM will not be fully visualized, and canaloplasty has to be done. This in turn may increase the operative type. In sharp contrast, the endoscope brings the surgeons eye to the tip of the scope. The wide angle of zero degree scope visualizes the entire TM. There is no need to frequently adjust the patients head or to do canaloplasty thereby saving operative time.

\section{Postoperative morbidity}

The postoperative morbidity of the surgery was greater in the group of the patients undergoing microscope assisted myringoplasty this was evaluated interms of postoperative scar. The patients undergoing microscopic assisted myringoplasty have more postoperative scar compared to endoscopic assisted type, as endoscopic assisted myringoplasty required small graft compared to MAM. In EAM small incision is given over hair line for temporalis fascia, which is progressively covered by hair. Rosens incision is given endaurally for elevating tympanomeatal flap.

\section{Discussion}

The main goals of treatment for COM are to relieve symptoms, rehabilitate hearing, and minimize complications and drainage. Conventionally, tympanoplasty is performed under an operative microscope. The main advantages of the microscopic approach are stereo vision and bimanual handling. However, despite providing direct exposure, microscopes require frequent adjustment and may still not be sufficient when encountering protruding structures, particularly the anterior wall. In a study conducted by Furukawa et al. ${ }^{10}$, the circumference of the perforation could not be confirmed with a microscope before denuding in $12.0 \%$ of cases. Furthermore, the entire perforation was not visible in $20.0 \%$ of cases after refreshing the edges. In contrast, endoscopy can show the entire tympanic membrane in one field with clear visualization of the perforation edges, even when the ear canal is narrow or protruding. Using a thin, rigid endoscope, a surgeon can perform minimally invasive procedures with protection of the anatomy, which allows for functional reconstruction during surgery ${ }^{11}$. When exploring the middle ear, the endoscope approach can provide more information regarding the orifice of the tube, the incudostapedial joint, and the roundwindow niche, which are usually difficult to observe under an operating microscope.

The wide angle of scope brings the tympanic membrane in one plane, more over the image can be magnified by just getting close to the structures. Thus there is no need to frequently manipulate patients head and moreover canal plasty can be avoided. Similar observations were made in two separate studies by Tarabichi $\mathrm{M}^{12}$ and Usami S, lijima $\mathrm{N}$ et $\mathrm{al}^{13}$. Moreover with angled endoscopes it is possible to visualize other structures like round window niche, sinus tympani, anterior epitympanum, eustachian tube area, facial recess, which are difficult to visualize with microscope. The endoscope brings the surgeons eye to the tip of the scope. The wide angle of zero degree scope visualizes the entire TM. There is no need to frequently adjust the patients head or to do canaloplasty thereby saving operative time.

By avoiding postaural incision in endoscope group, there is less dissection of normal tissues, less intraoperative bleeding, less incidence of postoperative pain, and better cosmetic result. Avoiding postaural route also reduces the chance of auricular displacement and asymmetry of pinna.

The microscope characteristically allows a broad and excellent image quality, with a direct and stereoscopic visualization. However, there are inherent limitations to the equipment, such as: decrease of brightness proportional to the 
magnification; limitation of the surgical field-of-view, especially in narrow spaces such as the EAC and the middle ear. Endoscopes, in turn, provide in-depth images in narrow recesses, while maintaining brightness and allowing different visualization angles.

Disadvantages associated with the endoscope involve working with only one hand and the absence of stereoscopic view, which many authors consider crucial for otologic surgery. Other criticisms of endoscope use in otologic surgery refer to the delicate nature of the surrounding structures, with potential risk of mechanical and thermal trauma, and the space conflict in the surgical field between optical fibre and the surgical instrument. The learning curve is another determining factor of surgical outcomes with the use of endoscopes, as observed in sinonasal and skull base surgery.

In our study total of 100 patients were taken in which 50 patients undergone EAM and 50 patients undergone MAM.

Female to male ratio is $1.7: 1$.

Graft uptake status was compared between both procedures. At the end of 1 month, initially showed EAM having more success rate $(88 \%)$ as compared to MAM but by the end of 6 months MAM showed $88 \%$ compared to EAM $86 \%$ which implies there is no much difference in graft uptake in both procedures. The chi square test shows statistically not significant ' $p$ ' value $(>0.005)$

Audiological gain shows a mean gain of $9 \mathrm{~dB}$ in both procedures which also implies no much difference $(p>0.005)$

Whereas variables such as operative time and postoperative scar are comparatively less in EAM than MAM, which also depends on the operating surgeon and the expertise in handling the equipment and also on the patient conditions as in narrowed external auditory canal, it is difficult to use endoscopic surgery where as if anterior overhang if present requires drilling of the overhang which requires more time compared to endoscopic surgery.

\section{Conclusion}

Transcanal endoscopic myringoplasty is a safe and feasibleprocedure, with good success rates for tympanic perforation closure and recovery of hearing thresholds. It can beperformed and taught in academic services in otorhinolaryngology, in addition to conventionalsurgery using a microscope.

\section{Acknowledgements:}

We would like to thank all the study participants and the authors from where we have cited the references for publication of this article.

\section{References}

1. Schuknecht HF (1993) Pathology of the ear (2nd edn), Lea and Febiger. Philadelphia, USA, pp. 191-253.

2. Glasscock ME, Shambaugh GE (2003) Surgery of the Ear (5th edn), Pathology and clinical course of inflammatory diseases of the middle ear. 21: 428-429.

3. Buckingham RA (1992) Fascia and perichondrium atrophy in tympanoplasty and recurrent middle ear atelectasis. Ann Otol Rhinol Laryngol 101(9): 755-758.

4. Browning GG, Merchant SN, Kelly G. Chronic otitis media. In: Gleeson MD (Ed,). Scott-Brown's Otorhinolaryngology, (7th edn), Head and Neck Surgery 3: 3425.

5. El Guindy A (1992) Endoscopic transcanal myringoplasty. J Laryngol Otol 106(6): 493-495.

6. Harugop AS, Mudhol RS, Godhi RA (2008) A comparative study of endoscope assisted and microscope assisted myringoplasty. Indian J Otolaryngol Head Neck Surg 60(4): 298-302.

7. Coskun BU, Cinar U, Seven H, Ugur S, Dadas B (2006) The effects of incision types in myringoplasty operations on cosmetics. Eur Arch Otorhinolaryngol 263(9): 820-822.

8. Fisch U (2008) Tympanoplasty, mastoidectomy and stapes surgery, (2nd edn), Thieme, New York, USA, pp. 8-11.

9. Usami S, lijima N, Fujita S, Takumi Y (2001) Endoscope-assisted Myringoplasty. Otorhinolaryngology 63(5): 287-290.

10. Furukawa $T$, Watanabe $T$, Ito $T$, Kubota $T$, Kakehata $S$. Feasibility and advantages of transcanal endoscopic myringoplasty. Otol Neurotol 2014; 35: e140-5.

11. Aoki K. Advantages of endoscopically assisted surgery for attic cholesteatoma. Diagn Ther Endosc 2001; 7: 99-107.

12. Muazz Tarabichi. Endoscopic transcanal middle ear surgery. Indian Journal Of Otolaryngology and Head \& Neck Surgery 2010;62(l):6-24.

13. Usami S, lijima N, Fujita S, Takumi Y. Endoscopic Assisted myringoplasty. ORL J Otorhinolaryngol Relat Spec. 2001 Sep-Oct;63(5):287-90. 